\title{
Article
}

\section{PIV Measurements of Large-Scale Structures in a Drag-Reducing Channel Flow with Surfactant Additives}

\author{
Masaaki Motozawa ${ }^{\dagger}$, Takahiro WatanaBe, and Yasuo KaWAGUCHI \\ Department of Mechanical Engineering, Tokyo University of Science \\ 2641 Yamazaki, Noda, Chiba 278-8510, Japan \\ (Received : October 20, 2010)
}

\begin{abstract}
Particle image velocimetry (PIV) measurements were performed on drag-reducing channel flow with surfactant additives. In order to clarify the large-scale structures, the measurement field was set to a relatively large size (130 $\mathrm{mm}$ in the streamwise direction $\times 40 \mathrm{~mm}$ in the wall-normal direction). The surfactant used in this experiment was a cationic surfactant CTAC, and NaSal was added to the solution with the same weight concentration as counter ions. We examined the effects of Reynolds number ranging from 15000 to 30000 and concentration of CTAC which was set to $25 \mathrm{ppm}$ or $40 \mathrm{ppm}$ in the flow. Based on these measurements, two-dimensional two-point correlations were calculated to illustrate the characteristic structures. In drag-reducing flow, there were large-scale fluid lumps in the near-wall region. These structures extended toward the streamwise direction and were inclined at approximately 10 degrees to the channel wall. These characteristic structures were only observed in the case of high drag reduction rate and the tendency of these structures did not change with varying Reynolds number. It is thus considered that turbulent coherent structures change, and sweep and ejection which produce skin frictional drag are suppressed.
\end{abstract}

Key Words: Turbulent drag reduction / PIV / Surfactant solution / Channel flow

\section{INTRODUCTION}

Some kinds of surfactant solutions significantly reduce skin frictional drag in turbulent flow even at rather dilute concentrations of tens of parts per million. This phenomenon was first reported by Toms in $1948^{1)}$ and thus came to be known as the Toms effect. Because turbulent drag reduction has the major benefit of reducing energy consumption in industrial applications, many successful applications for reducing drag have been developed. Takeuchi et al. ${ }^{2)}$ applied the drag-reduction effect with surfactant additives to a central heating/cooling system, and reported that pumping energy consumption was reduced by reached $65 \%$ in winter heating and by $47 \%$ in summer cooling.

Many researchers have investigated the drag-reducing effect by conducting experiments and numerical simulations. Warholic et al. ${ }^{3)}$ investigated the turbulence statistics of polymer homogeneous drag-reducing flow in a twodimensional channel by using PIV; they reported that the root mean square (RMS) of streamwise velocity fluctuation

\footnotetext{
$\dagger$ Corresponding author: Masaaki Motozawa

Department of Mechanical Engineering, Tokyo University of Science

2641 Yamazaki, Noda, Chiba 278-8510, Japan

Tel: +81-(0)4-7124-1501 (ext: 3963), E-mail: motozawa@rs.noda.tus.ac.jp
}

increased, that of wall-normal velocity fluctuation decreased, and Reynolds shear stress almost disappeared in dragreducing flow. There have also been some DNS studies on drag-reducing flow using constitutive equation models such as the Finitely Extensible Nonlinear Elastic with the Peterlin approximation (FENE-P) model ${ }^{4)}$ and Giesekus model ${ }^{5)}$ which express the properties of non-Newtonian fluid flow. They discussed high-order turbulence statistics and budgets of kinetic energy, which are difficult to obtain by experiments. Shikata et al. ${ }^{6}$ reported that the Maxwell model provides a close fit to $G^{\prime}$ and $G^{\prime \prime}$ data obtained from surfactant solutions; this model is very useful for expressing a viscoelastic fluid. However, there is uncertainty about shear-thinning in the surfactant solution, and the actual mechanism of drag reduction has yet to be fully explained. Therefore, it is worthy to investigate coherent structures in viscoelastic fluid flow experimentally.

In our previous study, we performed two-dimensional two-component PIV measurements in the $x-y$ plane $^{7)}$ and in the $x-z$ plane ${ }^{8)}$, and concluded that characteristic large-scale structures exist in drag-reducing flow. To investigate these large-scale structures, in this study we measured instantaneous components of streamwise velocity $u$ and wall-normal velocity $v$ of the $x-y$ plane by using the PIV system in a 
larger measurement field than that of our previous study. The measurement field was set to $130 \mathrm{~mm}$ (streamwise direction) $\times 40 \mathrm{~mm}$ (channel height). Based on these measurements, we discussed the characteristic turbulent structures of nonNewtonian fluid flow focusing on turbulence statistics and instantaneous velocity fields.

\section{EXPERIMENTAL APPARATUS}

Figure 1 shows the experimental apparatus employed in this study. The PIV measurements were performed on a closedcircuit water loop having a two-dimensional channel as a test section. This channel was made of transparent acrylic resin, with dimensions of $6000 \mathrm{~mm}$ length, $500 \mathrm{~mm}$ width and $40 \mathrm{~mm}$ height $(2 \delta)$. A honeycomb rectifier was set at the channel entrance to remove large eddies. In order to measure the flow rate $Q$, an electromagnetic flowmeter with an accuracy of $\pm 0.01 \mathrm{~m}^{3} / \mathrm{min}$ was installed in the flow path. Bulk mean velocity $U_{b}$ is estimated from $Q / A$, where, $A$ is the crosssectional area in the channel. The storage tank in the flow path contained a heater and an agitator in order to adjust the temperature of the fluid, which could be maintained at $25^{\circ} \mathrm{C}$ with an accuracy of $\pm 0.1^{\circ} \mathrm{C}$. Two pressure taps were attached on one side of the channel wall at a distance of $1650 \mathrm{~mm}$. The static pressure gradient between these two taps was measured in order to estimate the drag reduction rate (DR). The friction coefficient is given by the following equation;

$$
C_{f}=\frac{\tau_{w}}{1 / 2 \rho U_{b}^{2}}
$$

DR was defined by Eq. (2) using the friction coefficient of water and drag-reducing flow;

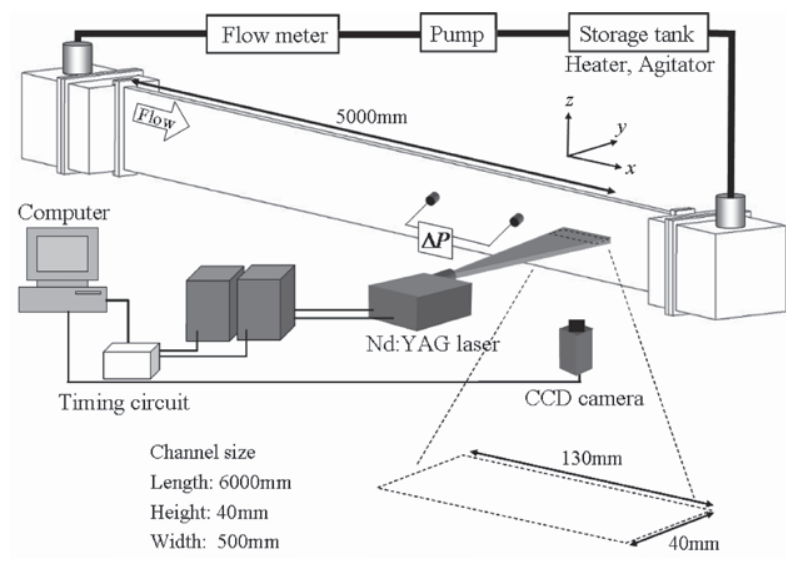

Fig. 1. Experimental apparatus.

$$
D R=\frac{C_{f, w}-C_{f, s}}{C_{f, w}} \times 100
$$

where, subscripts $w$ and $s$ represent water and surfactant solution, respectively.

The position of PIV measurements is located at $5000 \mathrm{~mm}$ downstream from the entrance of the channel. The surfactant used in this experiment was a cationic surfactant, cetyltrimethyl ammonium chloride (CTAC: $\mathrm{C}_{16} \mathrm{H}_{33} \mathrm{~N}\left(\mathrm{CH}_{3}\right)_{3} \mathrm{Cl}$ ). Local tap water was used as a solvent. Sodium salicylate (NaSal) was added to this surfactant solution in order to provide counter ions. The concentration of surfactant solution was $25 \mathrm{ppm}$ or $40 \mathrm{ppm}$. The molar ratio of counter ions to surfactant was $\xi=2$. Reynolds number was defined as;

$$
\operatorname{Re}=\frac{U_{b} \cdot H}{v}
$$

where, $H$ is the height of the channel and $v$ is the kinematic viscosity of the solvent. Re ranged from 15000 to 30000 for each case.

The double-pulse laser (New Wave Research Co. Ltd., MiniLase $30 \mathrm{hz}$ ) was a combination of a pair of $\mathrm{Nd}$ :YAG lasers, each having an output of $30 \mathrm{~mJ} /$ pulse and wavelength of $532 \mathrm{~nm}$. The laser sheet thickness and spread angle were set to $1 \mathrm{~mm}$ and $20^{\circ}$, respectively. The CCD camera (Dantec Dynamics Co. Ltd., Flowsense 4M) had a resolution of $2048 \times 2048$ pixels. The flow was seeded by nylon powder having a nominal diameter of $4.1 \mu \mathrm{m}$ as tracer particles. The measurement field was set to $130 \mathrm{~mm}$ (streamwise direction) $\times 40 \mathrm{~mm}$ (channel height). We obtained $125 \times 70$ vectors for each direction in the instantaneous velocity fields. Turbulence statistics were calculated from 500 velocity vector fields.

\section{RESULTS AND DISCUSSIONS}

\subsection{Drag Reduction Rate}

Table I shows the drag reduction rate for each experimental condition. The drag reduction rate increases with increasing Reynolds number except for $25 \mathrm{ppm}$ at $\mathrm{Re}=30000$, and a maximum drag reduction of $65 \%$ was obtained. In general,

Table I. Drag reduction rate.

\begin{tabular}{|c|c|c|}
\hline Reynolds number & $25 \mathrm{ppm}$ & $40 \mathrm{ppm}$ \\
\hline 15000 & $50 \%$ & $35 \%$ \\
\hline 20000 & $55 \%$ & $49 \%$ \\
\hline 25000 & $58 \%$ & $58 \%$ \\
\hline 30000 & $8 \%$ & $65 \%$ \\
\hline
\end{tabular}


drag reduction is related to the existence of a thread-like micelle network in the surfactant solution. Wunderlich et $a l^{9)}$ reported that long thread-like micelles form under high shear rate in the surfactant solution and a shear induced state (SIS) occurs as a non-Newtonian fluid behavior. In general, thread-like micelles are formed under suitable conditions of steady shear, surfactant concentration and temperature. However, because the concentration of CTAC solution used in this experiment was very dilute, we could not measure rheological parameters such as shear viscosity and relaxation time. Tamano et al. ${ }^{10)}$ measured the shear viscosity of $65 \mathrm{ppm}$ and 100 ppm CTAC solutions by a capillary viscometer, and confirmed that SIS occurred in these dilute CTAC solutions and that SIS is closely related with drag reduction. In our experiment, although we performed experiments with more dilute surfactant solutions, the viscoelasticity of the surfactant solution seemed to increase with the transition to SIS and a high drag reduction rate was obtained.

On the contrary, in the case of $25 \mathrm{ppm}$ at $\mathrm{Re}=30000$, the thread-like micelle network was broken by high mechanical shear stress and so the micelle structure did not form in this condition. As a result, the drag reduction rate was too small compared with the other cases.

\subsection{Turbulence Statistics}

Figure 2 shows the distribution of RMS of streamwise velocity fluctuation of water flow and drag-reducing flow of 25 ppm CTAC, normalized by the friction velocity $u_{\tau}$. The horizontal axis is normalized by half of the channel height $\delta$. In drag-reducing flow, although the streamwise velocity fluctuation has almost the same value near the channel wall, it sharply decreases toward the center of the channel. As a result, $u^{\prime}{ }_{r m s}$ of drag-reducing flow is smaller than that of water flow

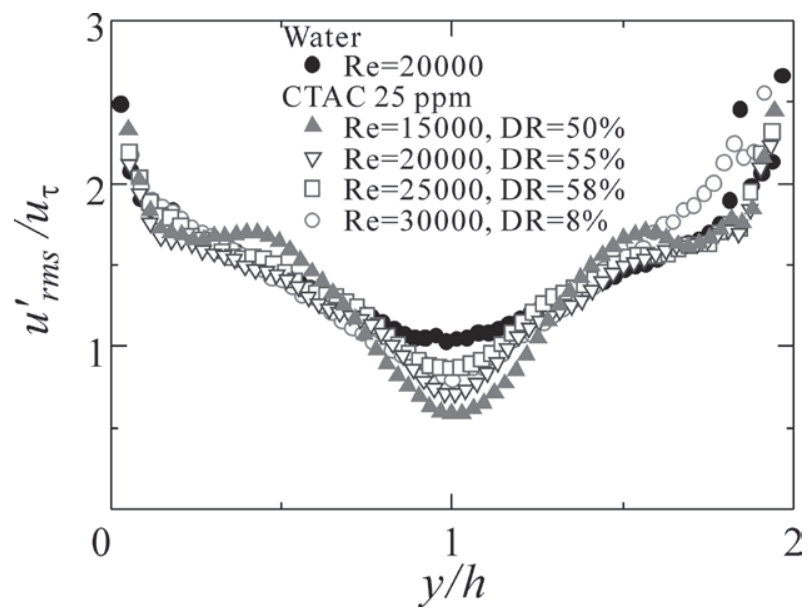

Fig. 2. RMS of streamwise velocity fluctuation. at the center of the channel. In addition, although the result is not shown in this paper, the RMS of wall-normal velocity fluctuation in the drag-reducing flow is smaller than that of the water flow throughout the channel; this tendency clearly appeared in the case of drag-reducing flow.

Figure 3 shows the Reynolds shear stress of water flow and surfactant solution flow. In the case of drag-reducing flow with high drag reduction rate, Reynolds shear stress is almost zero throughout the channel. This indicates that the momentum transport mechanism of drag- reducing flow is quite different from that of Newtonian fluid flow. A similar tendency of the change in turbulence statistics was also obtained for the 40 ppm CTAC.

The viscoelastic effect of surfactant solution also appears to increase the velocity fluctuation. Actually, in the drag-reducing channel flow with surfactant solution, the correlation between streamwise velocity fluctuation $u$ ' and wall-normal velocity fluctuation $v$ ' decreases and Reynolds shear stress $-\overline{u ’ v}$ ' also decreases due to the effect of surfactant solution as shown in Fig. 3. However, $u$, normalized by friction velocity $u_{\tau}$ of the drag-reducing flow slightly increases compared with water flow (see Fig. 2) at $y / \delta$ $=0.5$. This indicates that viscoelasticity increases the intensity of fluctuation in the drag-reducing surfactant channel flow. A similar result of an increase in fluctuation was reported in turbulent boundary layer experiments with surfactant solution conducted by Tamano et al. ${ }^{10)}$

In this study we also performed experiments with two concentrations of CTAC solution (25 ppm and $40 \mathrm{ppm}$ ) while varying the Reynolds number from 15000 to 30000 by changing the bulk mean velocity. Therefore, an increase of Reynolds number corresponds to an increase of Weissenberg number defined by relaxation time and friction velocity.

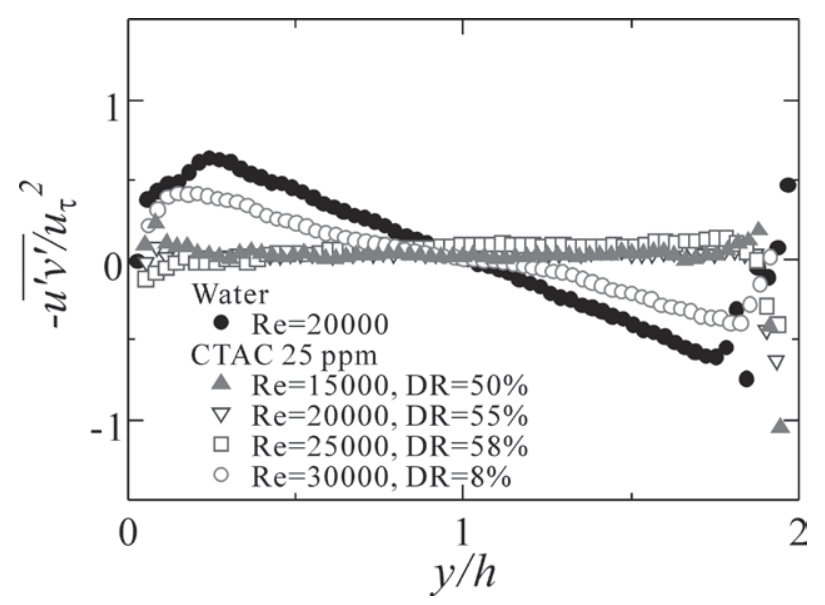

Fig. 3. Reynolds shear stress. 
Ishigami et al. ${ }^{11)}$ and Yu et al. ${ }^{12)}$ carried out a DNS study of drag reducing-flow using the Giesekus model for surfactant solution and investigated the relation between Weissenberg number and drag reduction. According to their calculations, high drag reduction can be achieved by suppressing the production of turbulence for high Weissenberg number. Therefore, our experimental results match those of their DNS study.

\subsection{Discussion of Large-scale Structures}

In order to investigate turbulent structures in detail in the near-wall region, we calculated two-dimensional two-point correlations for water flow and for surfactant drag-reducing flow. Two-dimensional two-point correlations are much useful for illustrating large-scale structures statically in the flow, and enable us to discuss the scale and angle of these structures from the calculations. The two-dimensional two-point correlation is defined by;

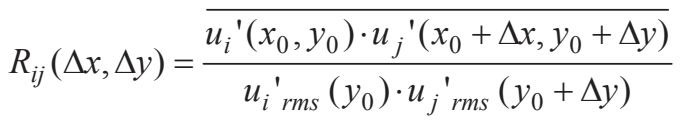

Figure 4 shows the contour map of $R_{u u}$ and $R_{u v}$ of water flow and drag-reducing flow at $x_{0} / \delta=250$ and $y_{0} / \delta=0.25$. As shown in Fig. 4(a), large fluid lumps, which have positive velocity fluctuation and negative velocity fluctuation, alternately appear in the water flow. These fluid lumps are approximately $x / h=2.0$ in length and $y / h=1.0$ in height. Kawamura et $a l .{ }^{13)}$ also reported these large-scale structures in Newtonian fluid turbulence by DNS. However, in dragreducing flow, these large-scale fluid lumps clearly appear only in the near-wall region (i.e. $0<y / \delta<0.5$ ). In addition, these structures extend toward the streamwise direction and are inclined at approximately 10 degrees to the channel wall as shown in Fig. 4(b). The scale of the streamwise direction is almost $x / h=3.0$. These large-scale structures did not appear only under the condition of 25 ppm CTAC solution at $\mathrm{Re}=$ 30000 , because the drag reduction rate is very small (about $8 \%$ ) in this condition. On the contrary, large-scale structures clearly appeared under all other conditions. Comparing the detailed structure for the same concentration of CTAC solution, the scale of these inclined fluid lumps and the angle to the channel wall are not so different at each Reynolds number. Therefore, this structure appears in the drag-reducing flow with high drag reduction rate and does not depend on Reynolds number.

Next, we discuss the relation between large-scale structures and rheological parameters. Table II comprehensively lists the relations among size and inclination angle of large-scale structures, Reynolds number and rheological parameters. In this table, $L_{x}$ is the streamwise size of large-scale structures estimated from Fig. $4, \delta$ is half of the channel height and $\lambda$ is the relaxation time of surfactant solution. In our experiment, (a)

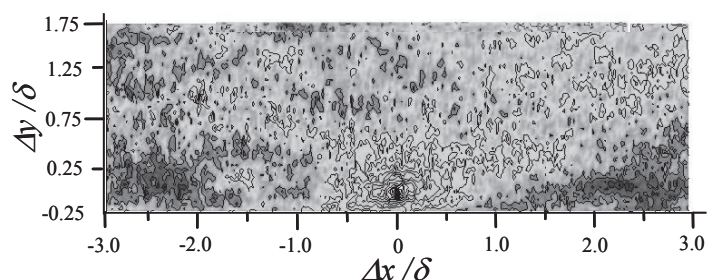

(b)

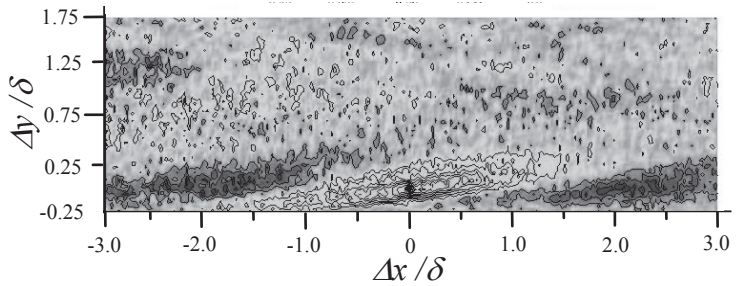

(c)

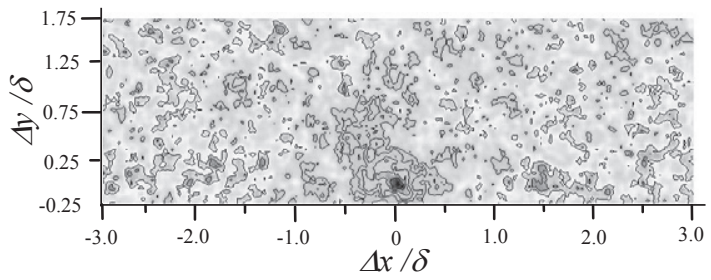

(d)

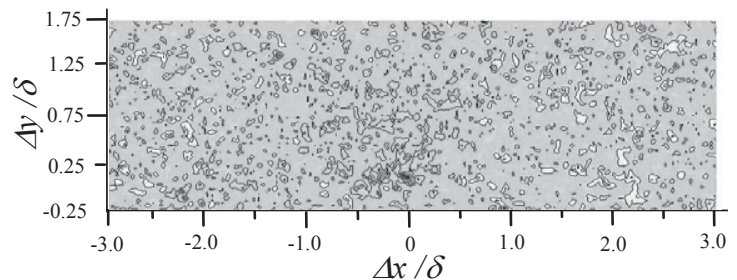

$\begin{array}{lllll}-0.32 & -0.2 & -0.08 & 0.04 & 0.16\end{array}$

Fig. 4. Two-dimensional two-point correlation function for $\mathrm{Re}=20000$ at $y / h=0.25$;

(a) $R_{u u}$ for water, (b) $R_{u u}$ for CTAC $25 \mathrm{ppm}\left(\mathrm{DR}=55 \%\right.$ ), (c) $R_{u v}$ for water and (d) $R_{u v}$ for CTAC $25 \mathrm{ppm}(\mathrm{DR}=55 \%)$. 
since we could not measure the relaxation time, it is given tentatively as $1 \mathrm{~s}$ based on experiments conducted by Suzuki et al. ${ }^{14)}$ Suzuki et al. measured the relaxation time of 1000 ppm solution of other kinds of surfactant and obtained a relaxation time of about $10 \mathrm{~s}$, but because we used a more dilute surfactant solution, we assumed a relaxation time of $1 \mathrm{~s}$. This table clearly indicates that the size of the large-scale structures $\left(L_{x} / \delta\right)$ and inclination angle $(\theta)$ do not depend on the Reynolds number and rheological parameters $\left(L_{x} / \lambda u_{\tau}\right)$.

Figures 4(c) and (d) show $R_{u v}$ of water flow and that of drag-reducing flow, respectively. In the water flow, $R_{v \sigma}$ has a correlation, whereas in the drag-reducing flow there is almost no correlation because Reynolds shear stress in drag-reducing flow is almost zero.

Based on this analysis, we proposed a conceptual flow structure of drag-reducing flow. Figure 5 schematically illustrates the large-scale structures of Newtonian fluid flow and non-Newtonian fluid flow in the $x-y$ plane. In the case of water flow, Adrian et al. ${ }^{15)}$ proposed that a hairpin vortex head is observed as shown in Fig. 5(a). At the upstream of the

Table II. Relation among size and inclination angle of large-scale structures, Reynolds number and rheological parameters.

\begin{tabular}{c|ccccc} 
Concentration & $\operatorname{Re}$ & $L_{x} / \delta$ & $L_{x} / \lambda u_{\tau}$ & $\theta\left[{ }^{\circ}\right]$ & $\mathrm{DR}[\%]$ \\
\hline \multirow{5}{*}{ CTAC 25ppm } & 15000 & 2.9 & 4870 & 9 & 50 \\
& 20000 & 2.8 & 3830 & 9 & 55 \\
& 25000 & 2.9 & 3470 & 8 & 58 \\
& 30000 & - & - & - & 8 \\
\hline \multirow{5}{*}{ CTAC 40ppm } & 15000 & 3.1 & 4270 & 9 & 35 \\
& 20000 & 3.2 & 3910 & 9 & 49 \\
& 25000 & 3.1 & 3420 & 8 & 58 \\
& 30000 & 3.0 & 2710 & 8 & 65 \\
\hline
\end{tabular}

(a)

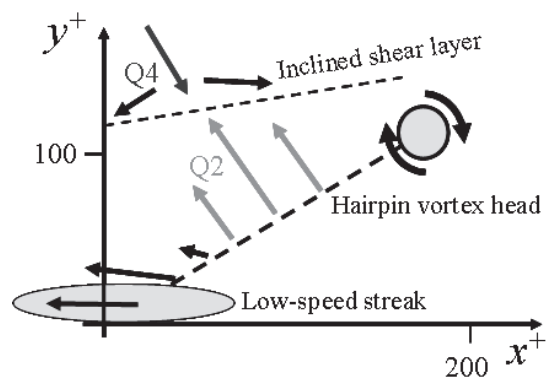

hairpin vortex head, strong ejection (Q2 motion) and sweep (Q4 motion) occur and these events contribute to increase the friction factor. An inclined shear layer is observed between these motions, and this structure is inclined at approximately 45 degrees to the wall. However, in the case of drag-reducing flow, ejection and sweep are largely suppressed, and so the hairpin vortex head is rarely observed in an instantaneous flow field. Instead of these coherent structures, large-scale inclined fluid lumps which have high velocity fluctuation and low velocity fluctuation are observed near the wall region as shown in Fig. 5(b). These structures are about two times larger than the hairpin vortex in the wall-normal direction.

Finally, we describe briefly the three-dimensional structure of the drag-reducing channel flow observed in this study and previous studies. ${ }^{7.8)}$ As shown in Fig. 2, there appeared an area where $u_{r m s}$ sharply decreased toward the center of the channel in the drag-reducing flow. For example, in the case of $\mathrm{Re}=$ 15000 , this area existed in the range of $y / \delta$ from 0.5 to 1.0. Therefore, two layers having different structures appear to coexist in drag-reducing channel flow.

In our previous experiment ${ }^{8}$, we measured the instantaneous velocity distribution in the $x-z$ plane by using PIV. This twolayer structure could be confirmed more clearly by calculating the vortex fluctuation. In the case of turbulent boundary flow, the characteristic distribution of $u_{r m s}$ was also observed in drag-reducing flow. ${ }^{16)}$

Moreover, the structure near the center of the channel has a grained structure, which is similar to the pattern of water flow. On the contrary, the structure in the near-wall region has a striped structure which extends in the streamwise direction and streaks of various lengths are observed. ${ }^{8)}$ The pattern is not so much different at other Reynolds numbers or concentrations. As discussed in this paper, large-scale structures exist in the layer of the near- wall region.

Fig. 5 Conceptual image of structure in the streamwise wall-normal plane; (a) Newtonian turbulent flow and (b) Non-Newtonian turbulent flow. 


\section{CONCLUSIONS}

The characteristic structures of drag-reducing flow in surfactant solution were investigated experimentally. We used PIV to measure instantaneous velocity fields in the $x-y$ plane on a relatively large size measurement filed and two-dimensional two-point correlations were calculated for analysing of the large-scale structures. The following conclusions in drag-reducing flow were obtained.

We performed experiments with dilute homogeneous CTAC solution (25 ppm and 40 ppm) and Reynolds number ranging from 15000 to 30000 . In the drag-reducing flow under these experimental conditions, sweep and ejection were greatly suppressed and large-scale inclined fluid lumps appeared alternately near the channel wall. On the contrary, these fluid lumps did not appear in the center of the channel. For the same CTAC concentration, the scale and inclination angle of these large-scale structures did not depend on Reynolds number and rheological parameters. These results were obtained only for high drag reduction rate.

\section{REFERENCES}

1) Toms BA, Proc. 1st Int Cong Rheology, 135 (1948).

2) Takeuchi H, Kawaguchi Y, Tokuhara K, Fujiwara Y, Proc 8th Int Conf Sustainable Energy Tech (2009)

3) Warholic MD, Heist DK, Katcher M, Hanratty TJ, Exp Fluids, 31, 474 (2001).
4) Dimitropoulos CD, Sureshkumar R, Beris AN, Handler RA, Phys Fluids, 13, 1016 (2001).

5) Yu B, Li FC, Kawaguchi Y, Int J Heat and Fluid Flow, 25, 961 (2004).

6) Shikata H, Hirata H, J Non-Newtonian Fluid Mech, 28, 171 (1988).

7) Gu W, Saito A, Motozawa M, Wang D, Kawaguchi Y, Proc 7th World Conf Exp Heat Transfer, Fluid Mechanics and Thermodynamics, 1025 (2009).

8) Watanabe T, Saito A, Motozawa M, Kawaguchi Y, Proc 6th Int Symp Turbulence, Heat and Mass Transfer, 947 (2009).

9) Wunderlich AM, Brunn PO, Colloid Polym Sci, 267, 627 (1989).

10) Tamano S, Itoh M, Inoue $T$, Kato K, Yokota K, Phys Fluids, 21, 045101 (2009).

11) Ishigami $T$, Tsukahara $T$, Kawaguchi $Y$, Yu $B$, Proc 6th Int Symp Turbulence and Shear Flow Phenomena (2009).

12) Yu B, Kawaguchi Y, Int J Heat and Fluid Flow, 24, 491 (2003).

13) Kawamura H, Abe H, Matsuo Y, Choi H, Proc Int Symp Dynamics and Statistics of Coherent Structure in Turbulence, 49 (2002).

14) Suzuki H, Fuller GG, Nakayama T, Usui H, Rheol Acta, 43, $232(2004)$

15) Adrian RJ, Meinhart CD, Tomkins CD, Journal of Fluid Mechanics, 422, 1 (2000).

16) Itoh M, Tamano S, Yokota K, Ninagawa M, Phys Fluids, 17, 075107 (2005) 